\title{
Improving Traffic Conditions by Using Neural Networks
}

Erick Cervantes C. ${ }^{1}$, José A. Castán R. ${ }^{2}$, Salvador Ibarra M. ${ }^{2}$, Julio Laria M. ${ }^{2}$, Javier Guzmán O. ${ }^{2}$, Mayra G. ${ }^{2}$, Treviño B. ${ }^{2}$ and Emilio Castán R. ${ }^{3}$

1. Computer Science, Engineering School, Autonomous University of Tamaulipas, Tamaulipas, Mexico

2. Computer Technology Group, Engineering School, Autonomous University of Tamaulipas, Tamaulipas, Mexico

3. Electric and Electronic Department, Madero Institute of Technology

\begin{abstract}
The paper promotes the implementation of a neural network approach to improve one of the most transcendent traffic conditions: the mobility of the cars in any particular junction. Neural networks have proven to be an effective paradigm of modern computing, providing extensive benefits in a wide range of applications. In this sense, the paper uses a BPNN (backpropagation neural network) model. The three input nodes are related to: $n 1$ : the amount of cars in the road; $n 2$ : the green light interval; and n3: the distance (taking into account the quantity of cars) between the first car in the intersection and the last car in the longest line in front of it. In particular, the paper promotes that each traffic light signal will be capable of offering a new green light interval according to the requirement and constrains of the vitality, ensuring a vehicular mobility level greater than $65 \%$. To assess this idea, the paper presents two experiments confronting the real world data versus experimental results. For example, in the first experiment, the BPNN improves the performance of the real data about vehicular mobility in almost $30 \%$. Finally, some conclusions and future work are presented.
\end{abstract}

Key words: Traffic conditions, neural networks, intelligent transport systems, vehicular mobility.

\section{Introduction}

In our days, the modern cities are facing to a set of well-defined challenges, among which must be highlighted the vehicular traffic control strategies. In fact, this challenge is one of the main concerns for the administrators of the cities because the failure of the scheduling in the traffic light signal control affects to all the entire population.

In this sense, the users are the main concern of the administrator, for thus, it is needed to identify a technology capable of being adjustable for the constraints and requirements of the roads. Some governments have approved some regulations that ensure benefits for the habitants. For example, in Mexico, the Communication and Transport Secretary proposes a standard capable of measuring the quality of the service offered by the traffic light signals. Such criterion is useful to the traffic experts in order to

Corresponding author: Salvador Ibarra M., Ph.D., research field: intelligent agents. identify the needs and opportunities areas of the traffic infrastructures. In this sense, intelligent systems have proven to be computational tools that cover substantially diverse research areas because of their decision-making paradigm based on reasoning processes. For instance, improving traffic-flow conduces to increase the service provided by the roads avoiding traffic jams and is reflected in a positive effect on the environment and the economy [1]. Some researchers have shown that these models produce realistic behavior [2-5]. According to this, the presented approach introduces a methodology capable of making flexible and autonomous decisions to adjust the green light interval of a traffic light signal, to the requirements of the road at any cycle of the phase. To assess the performance of the work, the paper presents two studied cases and their results.

\section{Related Work}

Current traffic controllers are based on microcontrollers and microprocessors. These traffic 


\section{Improving Traffic Conditions by Using Neural Networks}

light controllers have limitations because they do not have the flexibility of modification on a real time basis. In traffic signal control systems, detection of traffic variables at intersections is very important and it is the primary input data to determine signal timing. According to this approach, an application of fuzzy logic for multi-agent based autonomous traffic lights control using wireless sensors to overcome problems like congestion, accidents, velocity and traffic irregularities is presented in Ref. [2]. In fact, this approach provides a preferred solution by minimizing the vehicles waiting time, especially the emergency vehicles using fuzzy logic control systems under situations that normally occur during these situations. Fast transportation systems and rapid transit systems are nerves of economic development for any nation. All developed nations have well developed transportation systems with efficient traffic control in road, rail, and air. The monitoring and control of city traffic is one of the key issues, especially in metropolitan areas, considering the increasing number of vehicles and pedestrians. Numerous methods are available to reduce delays and the pollutant environmental problems caused by road traffic in major cities. Likewise, a new infrastructure to vehicle communication and control systems for intelligent speed control, which is based upon RFID (radio frequency identification) technology for recognition of traffic signals on the road, and high accuracy vehicles speed measurement with Hall Effect-Based Sensor is explained in Ref. [4]. To achieve a more efficient adaptation of the speed of the vehicle, the approach introduces an adaptation of a fuzzy logic controller based on sensor fusion of the information provided by the infrastructure installed on the road. Finally, the results suggest unexpected traffic circumstances and improve the safety of the occupants in the vehicle. In this light, an Intelligent Traffic Signal Controller using a FPGA controller based on a Neuro-Fuzzy system is presented in Ref. [6]. This approach is capable of making decisions to reduce delays at an intersection. In particular, the fuzzy logic theory in the controller provides an intelligent green interval response based on dynamic traffic using the input variables of the weighed traffic flow at the current and neighboring intersections. This paper argues that it is able to overcome the weakness of conventional traffic controllers by using its capability of providing a variety of green cycle intervals based on dynamic traffic load changes at every lane in a 4-way junction control. Roozemond [7] introduced an agent-based urban intersection control system that reacts to changes in the traffic environment and adapts itself to changing environments based on predefined rules. Besides the current trends to solve the computational methods, the development of the field simulated test environments to control and to optimize urban arterials is another interesting and promising area for further study. Nowadays there are computational tools that offer the opportunity of implementing and testing approaches in order to corroborate their ideas. Nonetheless these tools are not functional for all purposes. Because of this, some authors tend to develop their own tools, while they are generating their methodological proposals. For instance, a reservation-based approach to intersection control was designed and evaluated in Ref. [8]. This approach proposes the development of a novel simulator test bed for connected vehicle applications. Indeed, the test bed integrates a microscopic traffic simulator with a network simulator and an emission analyzer. Some experiments are presented using the developed simulator to compare the mobility and environmental benefits of the introduced approach against traditional control methods. Refs. [3, 9-15] are other examples of the aforementioned actions (see Table 1). To this end, a complete review of literature about agent-based approaches well suited to the domain of traffic and transportation system, because transportation systems are often geographically distributed in dynamics changing environments as presented in Ref. [16]. In particular, the paper reviews agent-based traffic 
Table 1 Example of a vehicular capacity in determined junction.

\begin{tabular}{|c|c|c|c|c|c|c|c|c|}
\hline HORA & $\mu_{\mathrm{L}}$ & $\mu_{\mathrm{M}_{\mathrm{A}}}$ & $\mu_{\mathrm{M} \mathrm{I}}$ & $\mu_{\mathrm{J}}$ & $\mu_{\mathrm{V}}$ & $\mu_{\mathrm{S}}$ & $\mu_{\mathrm{D}}$ & $\mu_{\mathrm{H}}$ \\
\hline $7-8$ & 93 & 88 & 74 & 81 & 93 & 28 & 5 & 66 \\
\hline $8-9$ & 83 & 79 & 81 & 64 & 80 & 20 & 5 & 59 \\
\hline $9-10$ & 66 & 67 & 52 & 52 & 58 & 31 & 7 & 48 \\
\hline $10-11$ & 67 & 63 & 73 & 58 & 60 & 43 & 26 & 56 \\
\hline $11-12$ & 61 & 74 & 71 & 61 & 63 & 30 & 25 & 55 \\
\hline $12-13$ & 87 & 77 & 75 & 84 & 71 & 46 & 61 & 72 \\
\hline $13-14$ & 108 & 88 & 107 & 88 & 91 & 37 & 55 & 82 \\
\hline$\mu_{\mathrm{D}}$ & 81 & 77 & 76 & 70 & 74 & 34 & 26 & \\
\hline
\end{tabular}

applications classified in five categories: (1) agent-based traffic control and management system architecture and platforms; (2) agent-based systems for roadway transportation; (3) agent-based systems for air-traffic control and management; (4) agent-based systems for railway transportation; and (5) multi-agent traffic modeling and simulation. However, software agents and artificial intelligence techniques in urban traffic systems have arisen as proper solutions in the last decade to provide high levels of efficiency in transport infrastructures. In despite of these efforts, the design, development and implementation of agent technology in traffic control systems are still immature and need further study in order to achieve accuracy and trustworthy applications to building traffic control systems relating to the control the demand levels and to minimize the pollutant emissions in urban traffic networks.

\section{Neural Network Model}

Long lines of vehicles, wide ranges of stop-times in intersections and environmental emissions are only some of the aspects that traffic control is looking forward to eliminate. Indeed there has been a growing interest among the transportation community in the uses of traffic simulation modeling for evaluating the systems performance of transportation networks [17]. In this sense, the paper proposes the implementation of a traffic light signal control based on BPNN (backpropagation neural network). The main idea argues that each intersection will be controlled by a BPNN capable of analyzing the requirements of each one of its roads, and using these data, be able to provide an adequate green light interval for each road. The following three nodes are proposed to the developed BPNN:

Node1: this node represents the amount of stopped cars in each road, calculated one second before the change of the signal from amber to green light. This information is generated for a simple model, which it is in-charge of simulate a normalized arrival of cars (1):

$$
\text { icar }_{k}=\left[\operatorname{abs}\left(r=[0: n] * \frac{m}{s}\right)\right]_{t_{a-1}}
$$

where $r$ is a value obtained from a monte-carlo table, which it is founded on a reliable identification of the random value devoted to a real world study and $\mu$ and $\sigma$ are the media and the standard deviation respectively.

In order to guarantee the reliability of the model, some experiments were ran, confronting the incoming volume of vehicles provided by the model versus the statistic data computed from an in-situ study of several intersections in Tampico, Tamaulipas, Mexico. Fig. 1 presents how the model results achieve a similar behavior than the data of the real world, during the execution of an experiment of 120 seconds. 
Improving Traffic Conditions by Using Neural Networks

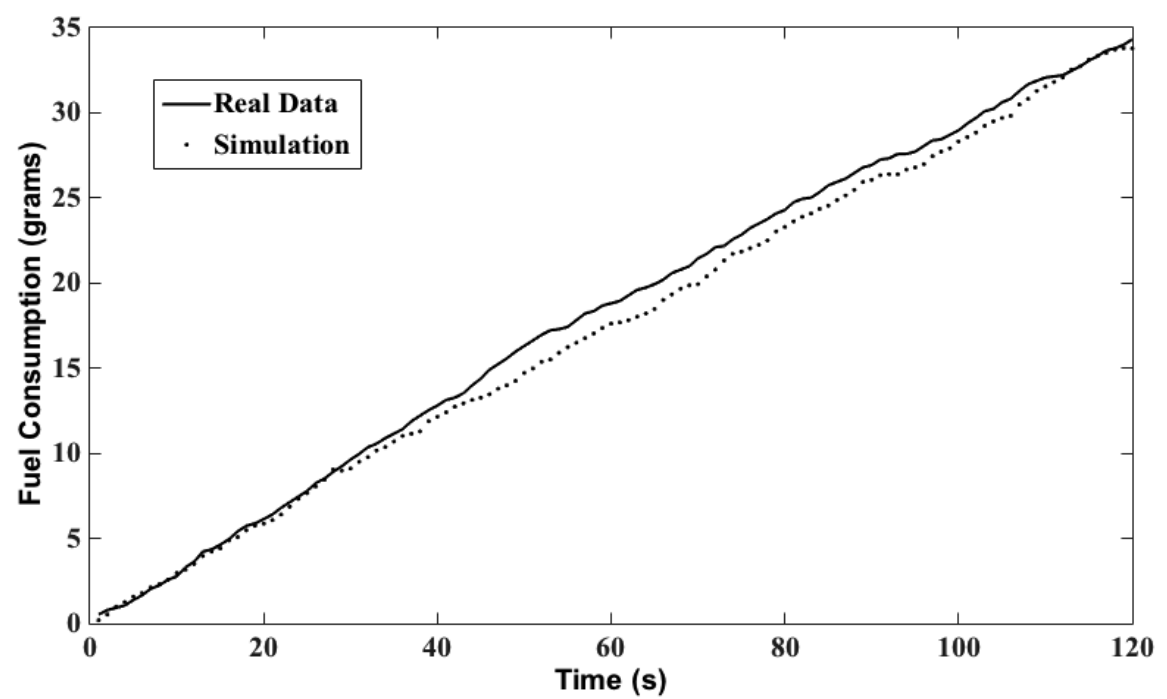

Fig. 1 Arriving car to a particular junction. Real data versus model results.

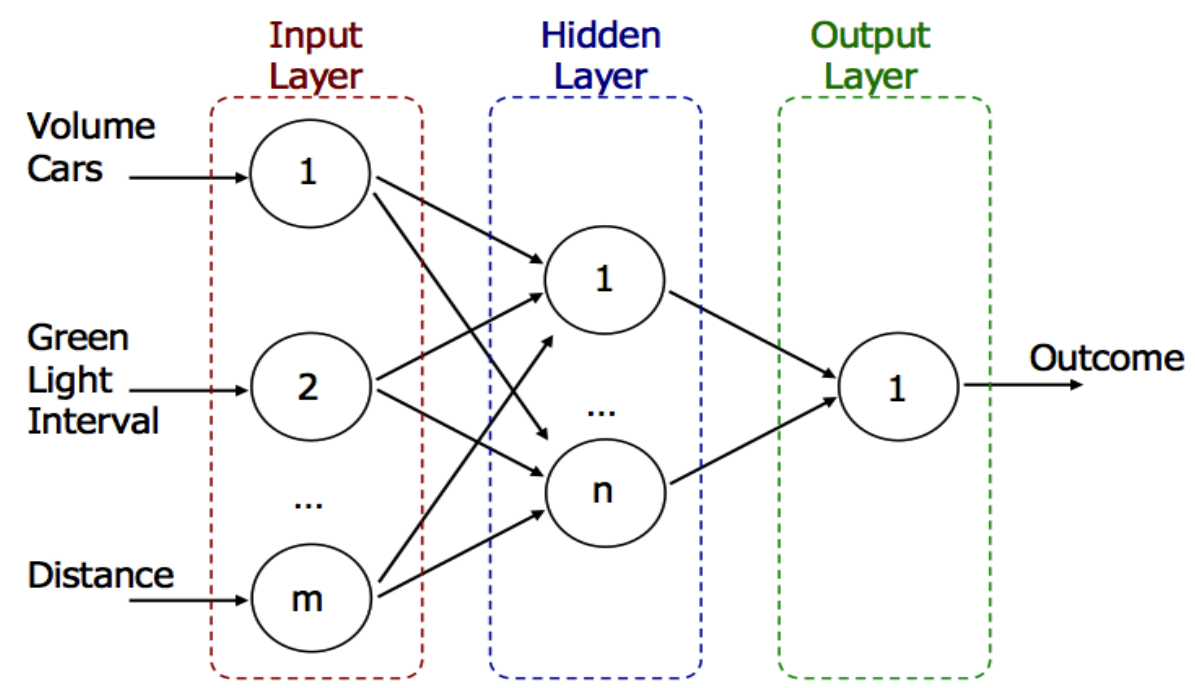

Fig. 2 Graphical representation of the proposed neural network.

Node2: for sake of simplicity, this node uses information provided by the system itself. It means, before the neural process, the system looks for the green light interval of the previous cycle. It is important to note, for the first iteration of each road, the BPNN uses information provided by the user of the system.

Node3: this input is related to the distance (the quantity of cars) between the first car of the studied intersection and the last car in the longer line at the follow line.

For the scope of this paper, only one intersection is considered and the rest of data is simulated in a controlled way for the system. In order to validate the accuracy of the BPNN during the training phase, Fig. 3 shows the performance of the model in 10,000 phases. It is noteworthy that 50,000 experiments were developed to ratify the reliability of the decision reached by the BPNN. Both the model based on BPNN and the parameters considered in the node inputs appear to be an approach according to solve the requirements of any intersection in a positive way. Finally, the performance of the BPNN does not improve after the phase 500, for thus, the number of experiments is fixed in 750 phases.

Output: a new green light interval, according to the 


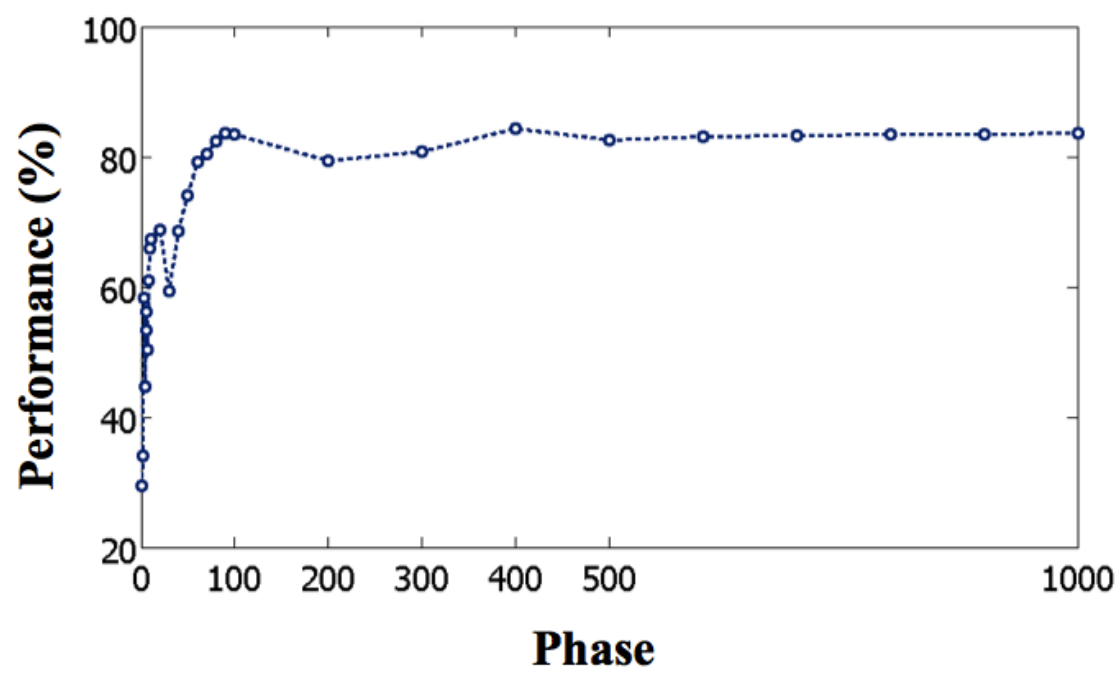

Fig. 3 BPNN training.

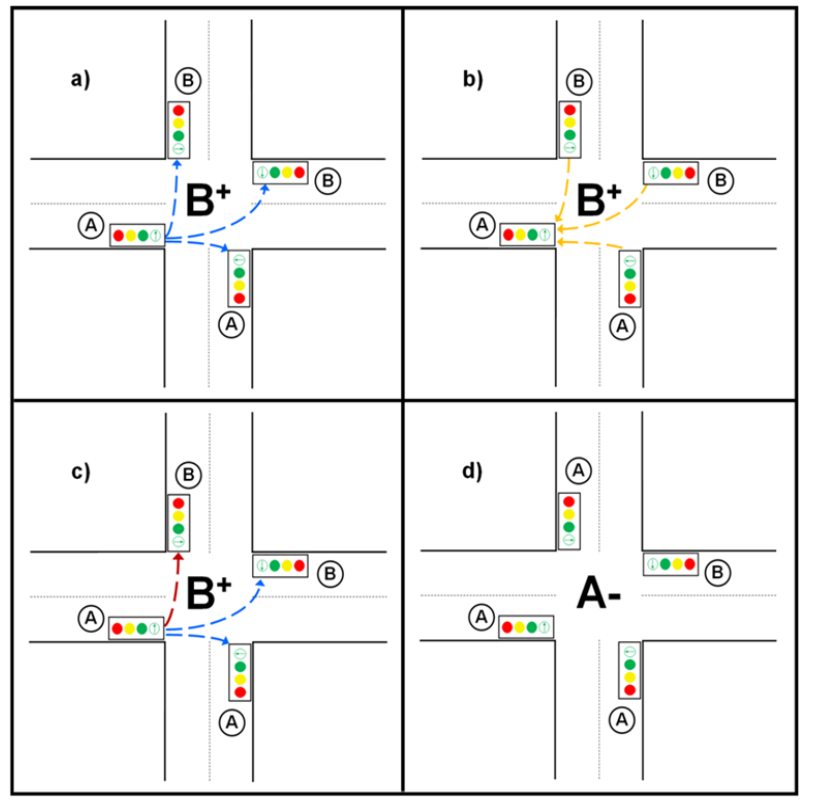

Fig. 4 General scheme of the overall system operation.

requirements of the road, with at least $61 \%$ of the vehicular mobility.

For example, in Fig. 4 is presented a scheme of the overall operation of the system when a light signal decides to modify its green light interval. First, the traffic light informs to the others traffic lights the performed modification in order to update the general phase of the junctions (see Fig. 4a). After that, the rest of traffic lights confirm the information and consider such information to modify the length of their red lights. To continue, the current traffic light calculates the new phase and advices this information to the next traffic light in order to corroborate the operation of the junction (see Fig. 4c). As we can see in Fig. 4, throughout the system operation, the quality of the intersection still does not improve $\left(\mathrm{B}^{+}\right)$. Once the traffic light performs the changes, obtained from the neural network process level the junction operability increases (A-) in a considerably way (see Fig. 4d).

\section{Experiments and Results}

In this section, two studied cases are presented, considering two of the most important roads of Tampico, Tamaulipas, Mexico. Vehicular mobility is one of the main targets for both experimental phases. The experiments are developed under four demand levels, proposed in: $50 \%, 100 \%, 150 \%$ and $200 \%$, which are simulated modifying the variable $r$ in the Eq. (1). Preliminary results of the model are compared against real data as well as versus results presented in Ref. [17].

Experiment 1. The relevance of this experiment is because two of the main roads of Tampico converge in this intersection (see Fig. 5). The initial data for the generation of the vehicle arrivals are presented in Table 2.

The performance is compared to demonstrate the benefits of the BPNN over traditional traffic control 
Improving Traffic Conditions by Using Neural Networks

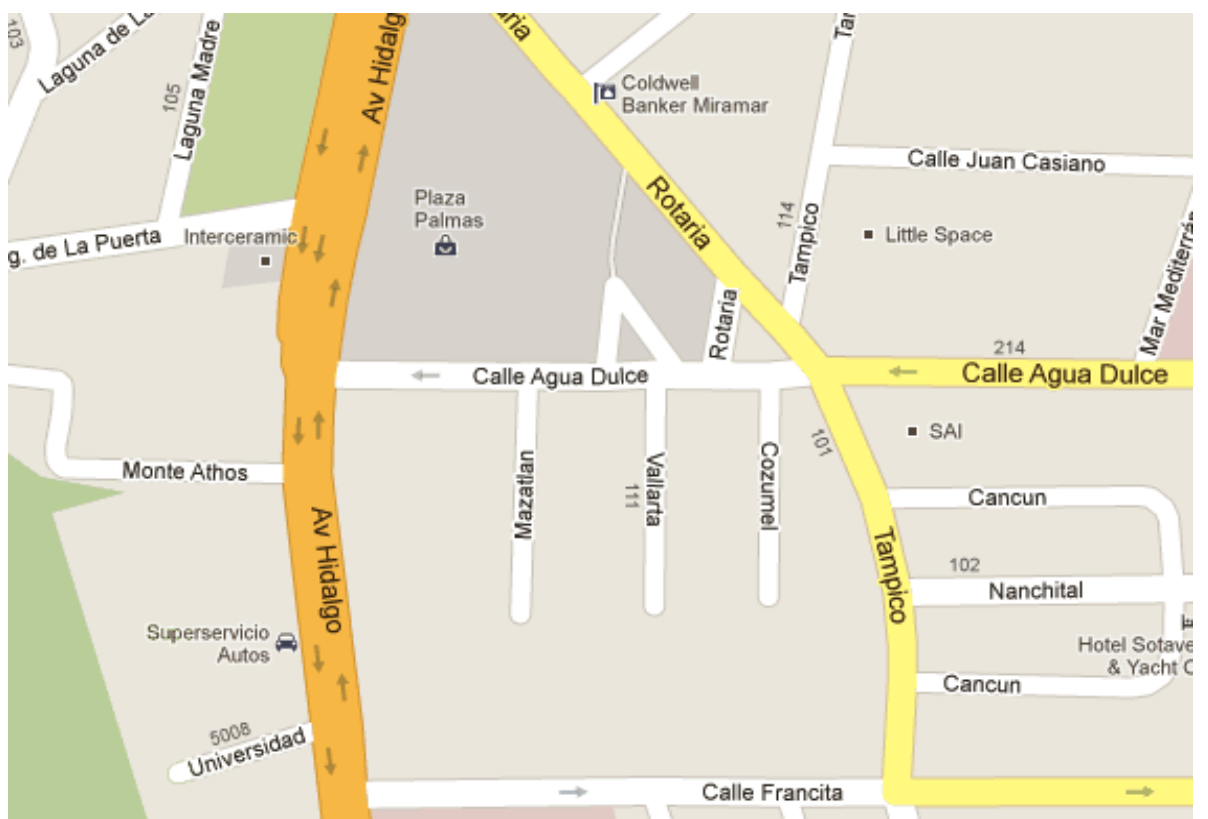

Fig. 5 Scenario 1 (Google Maps).

Table 2 Real data-Scenario 1.

\begin{tabular}{llll}
\hline Demand level & $r$ & $\mu$ & $\sigma$ \\
\hline $50 \%$ & {$[0-1]$} & & \\
$100 \%$ & {$[0-3]$} & 2.5 & 1.8 \\
$150 \%$ & {$[1-4]$} & & \\
$200 \%$ & {$[2-4]$} & & \\
\hline
\end{tabular}

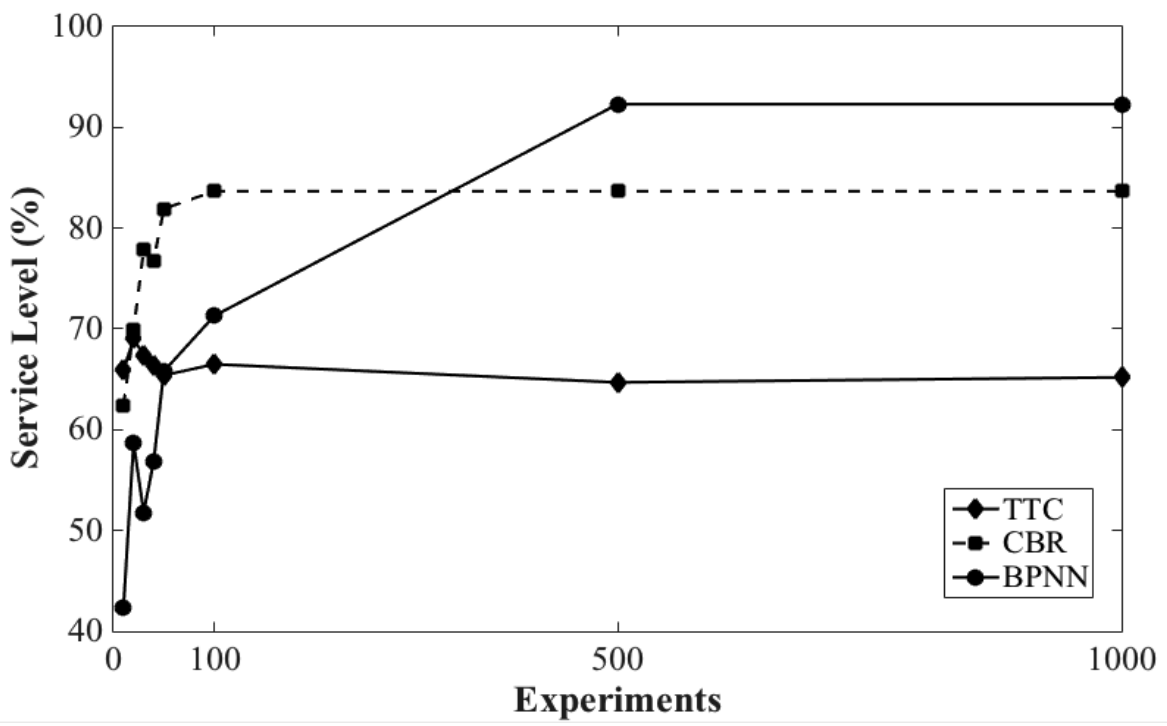

Fig. 6 Service level versus total of experiments.

TTC methods. In short, in Fig. 6 the trace of the results shows how the vehicular mobility is better than TTC in almost $30 \%$ and is better than CBR model in 9\%. The performance of BPNN appears to take certain amount of experiments before to confirm a stable behavior, unlike the CBR model (almost 220 proves). However, once the BPNN is stable, reaches a higher mobility level. Besides, Fig. 7 depicts the performance 


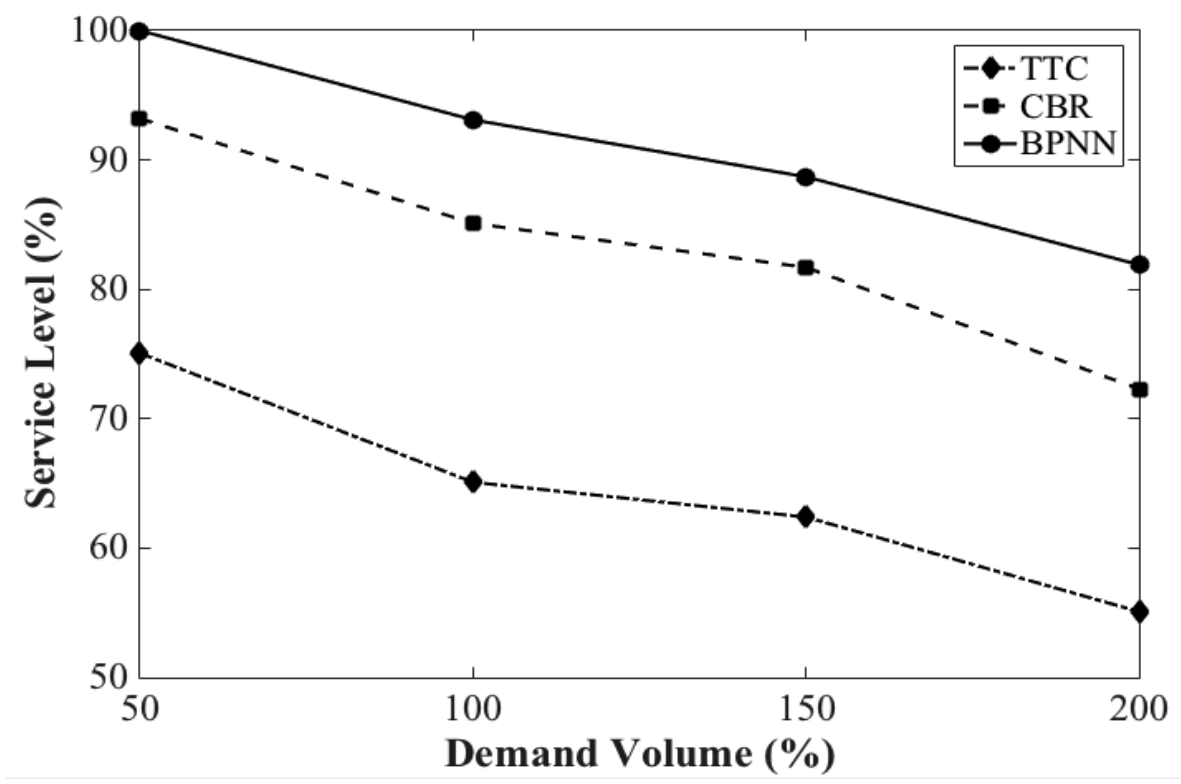

Fig. 7 Service level versus demand volume.

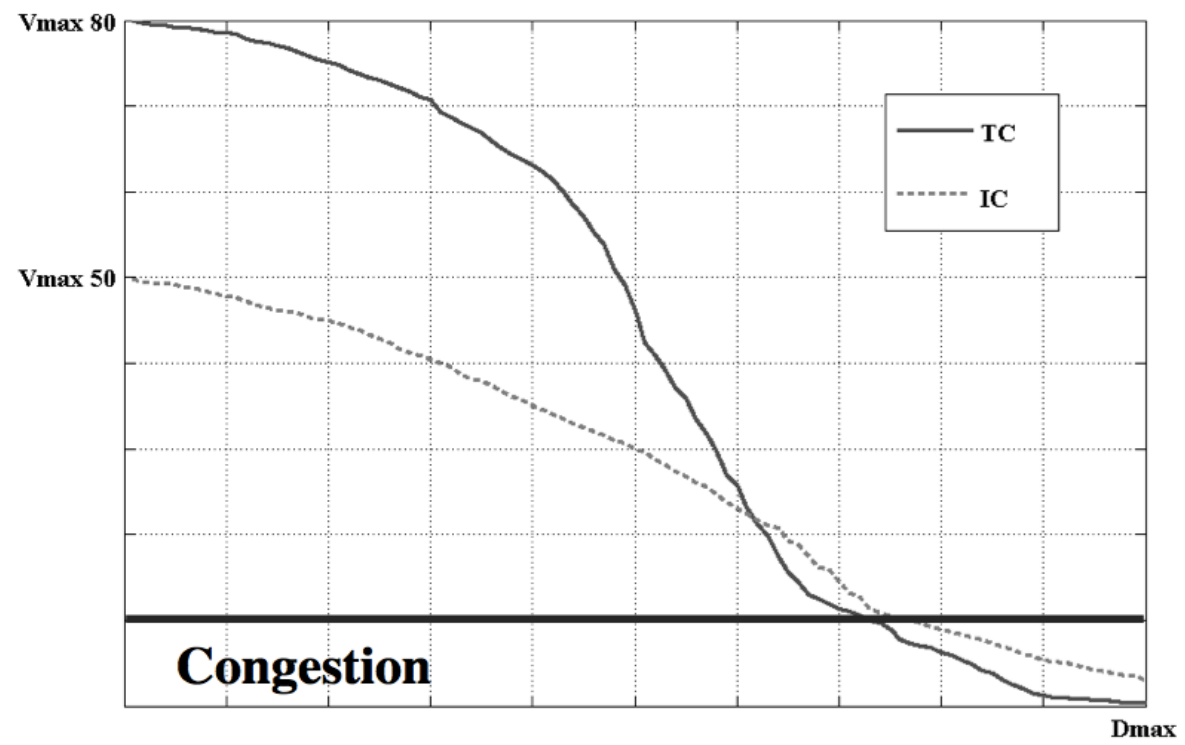

Fig. 8 Traffic velocity versus the traffic volume.

of the three models under four demand levels. In fact, the BPNN holds a better performance than the other models while they are under different demand levels.

In addition, Fig. 8 compares the traffic velocity against the traffic volume. This comparison identifies the advantages of the proposed methodology IC over traditional approaches TC to improve vehicular mobility under different types of volume demand.

Experiment 2. The second test (see Fig. 9) involves data of a real-world intersection, taking into account one of the most important roads in Tampico City, MX (Hidalgo Ave) and one street (Agua-Dulce St.). The data used in these experiments have been obtained in real traffic studies dating from June to July, 2015. In particular, the information for the incoming car simulation is presented in Table 2 .

The obtained results are compared in order to emphasize the advantages of traffic control based on BPNN over the pre-programming traditional control used in the synchronization phase of the studied 


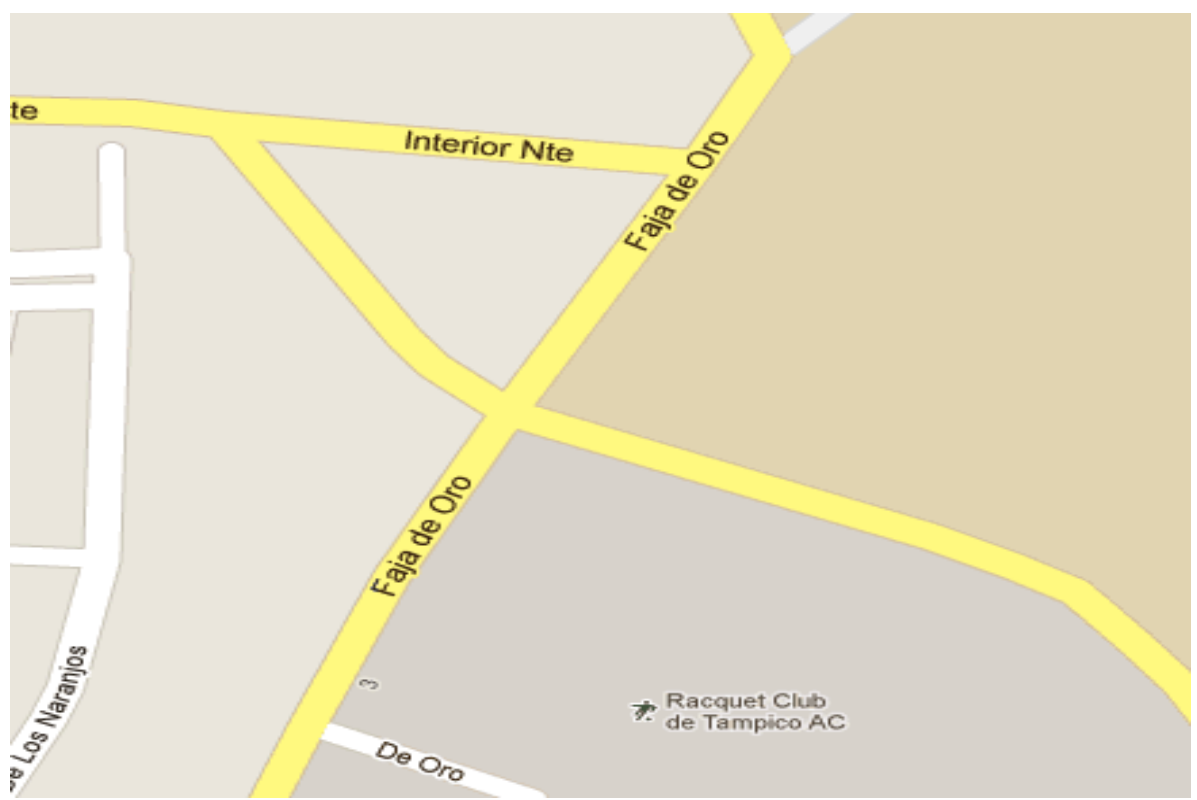

Fig. 9 Scenario 2 (Google Maps).

Table 2 Real Data-Scenario 2.

\begin{tabular}{llll}
\hline Demand Level & $r$ & $\mu$ & $\sigma$ \\
\hline $50 \%$ & {$[0-1]$} & & \\
$100 \%$ & {$[0-3]$} & 2.3 & 1.7 \\
$150 \%$ & {$[1-4]$} & & \\
$200 \%$ & {$[2-4]$} & & \\
\hline
\end{tabular}

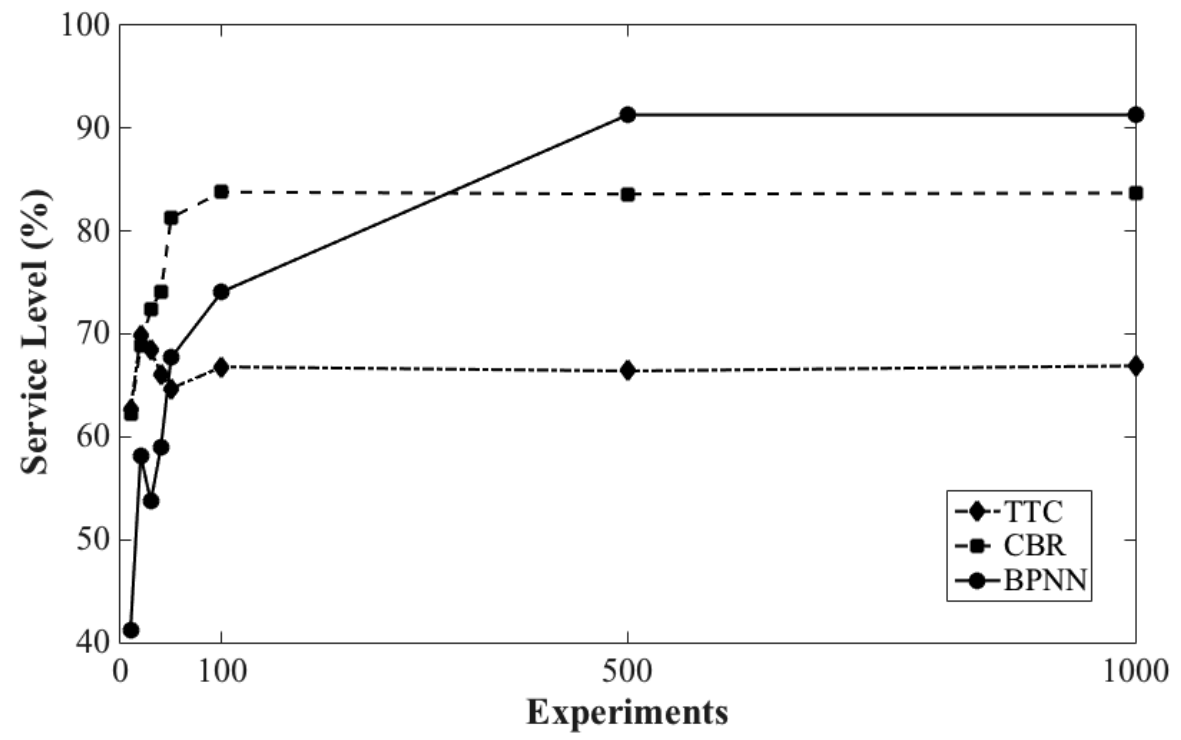

Fig. 10 Service level versus total of experiments.

intersection. To evaluate the decision-making process of the BPNN approach proposed in this work, Fig. 10 depicts how the BPNN model reaches a suitable behavior after 500 experiments along 1,000 trials.
Moreover, the BPNN reaches a better performance than the TTC and the CBR. In particular, the proposed method overcomes to the other two models in $8 \%$ and $29 \%$ respectively, in mobility aspects (see Fig. 11). 


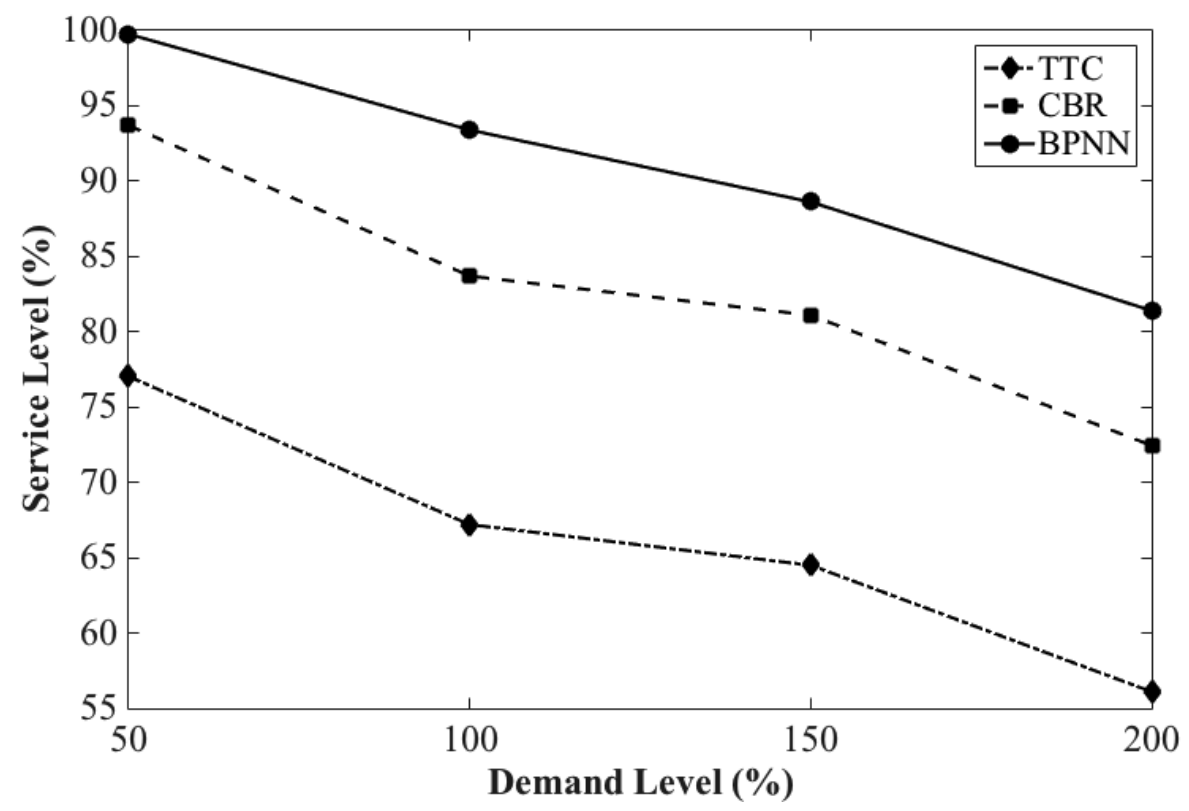

Fig. 11 Service level versus demand volume.

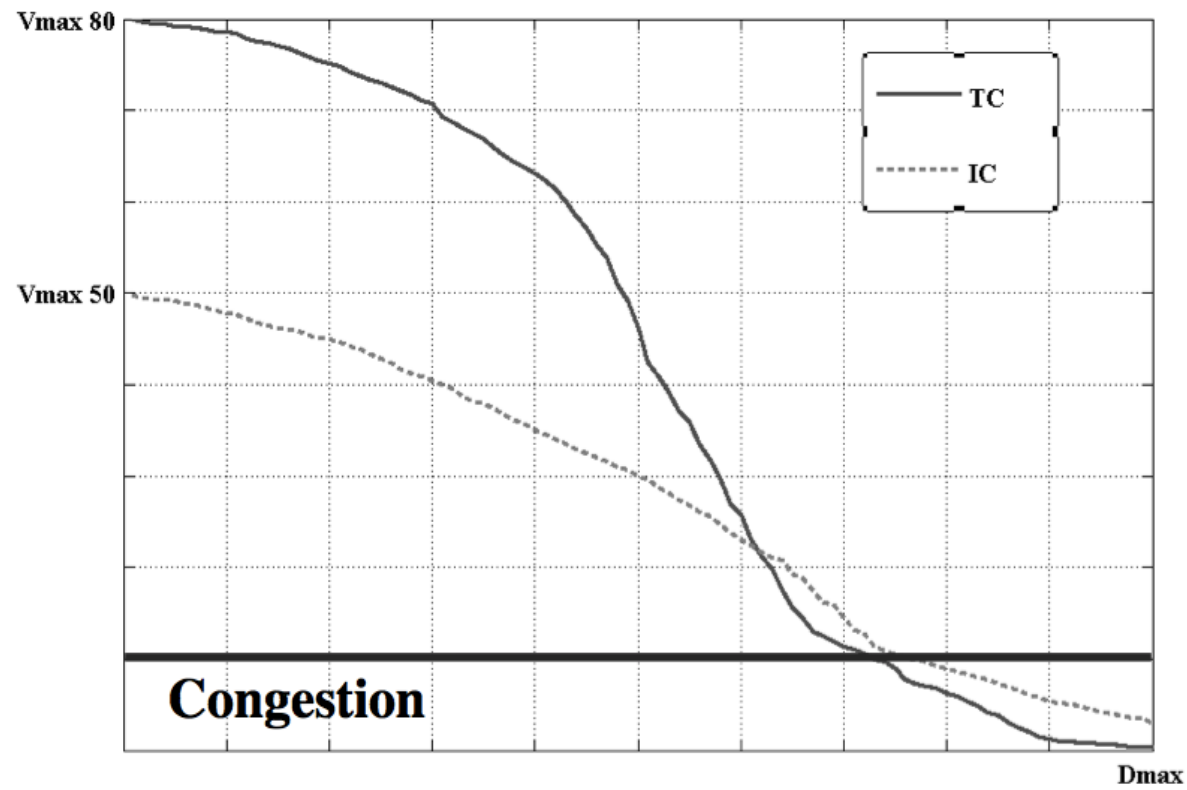

Fig. 12 Traffic velocity versus the traffic volume.

Besides, Fig. 12 compares the traffic velocity against the traffic volume. This comparison identifies the advantages of the proposed methodology over the traditional approach to improve vehicular mobility under different types of volume demand.

\section{Conclusions and Future Work}

Road traffic congestion is one of the main causes in low productivity and the decrease in modern city standards. Therefore, the use of the avenues and streets are a common way to transport activities in the daily life. Such consideration is a key factor to determine the quality of city services. Unfortunately, the uncontrolled growth of the volume of cars on the streets is a transcendental challenge because the implemented model to regulate the traffic uses 


\section{Improving Traffic Conditions by Using Neural Networks}

techniques that lack of a technological upgrading. For this reason, the paper introduces a novel model to manage traffic light signals using neural networks. Specifically, the model implements a backpropagation neural network approach in-charge of ensuring that at least $61 \%$ of vehicles are dispatched during a green light interval.

The presented study is used to evaluate the vehicular mobility in two different intersections, contrasting the experimental results versus traditional traffic control and a CBR methodology. The reported results show how BPNN reaches a better performance than the other two methods in both experimental phases.

Intelligent traffic lights using sophisticated computational algorithms represent a novel futuristic approach to autonomous intersections control. Future research should focus on assessing the reliability, robustness and safety of the approach under a wide range of expected operating conditions and failure modes. In spite of this, there are some limitations about the scope of this work. For example, the adaptation of other automatic learning techniques in order to evaluate the effectiveness of these promising approaches.

\section{Acknowledgment}

This paper was supported by the Secretaría de Investigación y Posgrado at the Universidad Autónoma de Tamaulipas, Mexico, by the project PFI2014-59.

\section{References}

[1] Wen, W. 2008. "A Dynamic and Automatic Traffic Light Control System for Solving the Road Congestion Problem." Expert Systems with Applications 34 (4): 2370-81. [doi:/10.1016/j.eswa.2007.03.007].

[2] Saeed, Y., Khan, S., Ahmed, K., and Mubashar, S. M. 2011. "A Multi-Agent Based Autonomous Traffic Lights Control System Using Fuzzy Control." International Journal of Scientific \& Engineering Research 2 (6), June-2011 1, ISSN 2229-5518.

[3] Chen, B., and Cheng, H. 2010. "A Review of the
Applications of Agent Technology in Traffic and Transportation Systems." IEEE Transactions on Intelligent Transportation Systems $11 \quad$ (2). [doi:10.1109/TITS.2010.2048313].

[4] Pérez, J., Seco, F., Milanés, V., Jiménez, A., Díaz, J. C., and De Pedro, T. 2010. "An RFID-Based Intelligent Vehicle Speed Controller Using Active Traffic Signals." Sensors 10 (6): 5872-87. [doi:10.3390/s100605872].

[5] Ngai, E., and Riggins, F. 2008. "RFID: Technology, Applications, and Impact on Business Operations." International Journal of Production Economics 112 (2). [doi: science/journal/09255273/112/2].

[6] Zade, A. R., and Dandekar, D. R. 2011. "FPGA Implementation of Intelligent Traffic Signal Controller Based on Neuro-Fuzzy System." International Conference on Advanced Computing, Communication and Networks.

[7] Roozemond, D. A. 2001. "Using Intelligent Agents for Pro-Active, Real-Time Urban Intersection Control." European Journal of Operational Research 131 (2): 293-301. [doi: science/article/pii/S0377221700001296].

[8] Huang, S., Sadek, A., and Zhao, Y. 2012. "Assessing the Mobility and Environmental Benefits of Reservation-Based Intelligent Intersections Using an Integrated Simulator." IEEE Transactions on Intelligent Transportation Systems.

[9] Chen, R. S., Chen, D. K., and Lin, S. Y. 2005. “ACTAM: Cooperative Multiagent System Architecture for Urban Traffic Signal Control." In Proc. of the IEICE Trans. Inf. Syst. E88-D: 119-26.

[10] Garcia-Serrano, A. M., Teruel, D., Carbone, F., and Méndez, V. 2003. "FIPA-Compliant MAS Development for Road Traffic Management with a Knowledge-Based Approach: The TRACK-R Agents." In Proc. of Challenges Open Agent Syst. Workshop, Melbourne, Australia.

[11] Hernandez, J. Z., Ossowski, S., and Garcia-Serrano, A. 2002. "Multiagent Architectures for Intelligent Traffic Management Systems." Transportation Research Part C: Emerging Technologies 10: 473-506. [doi: science/article/pii/S0968090X02000323].

[12] Liu, Z. Q., Ishida, T., and Sheng, H. Y. 2005. "Multiagent-Based Demand Bus Simulation for Shanghai." In Proc. Massively Multi-Agent Syst. I 3446: 309-22.

[13] Wang, F. Y. 2005. "Agent-Based Control for Networked Traffic Management Systems." IEEE Intelligent Systems 20 (5): 92-6. [doi: 10.1109/MIS.2005.80].

[14] Wang, F. Y. 2008. "Toward a Revolution in Transportation Operations: AI for Complex Systems." IEEE Intelligent Systems 23 (6): 8-13. [doi: 10.1109/MIS.2008112].

[15] Zhang, H. S., Zhang, Y., Li, Z. H., and Hu, D. C. 2004. 


\section{Improving Traffic Conditions by Using Neural Networks}

"Spatial-Temporal Traffic Data Analysis Based on Global Data Management Using MAS." IEEE Transactions on Intelligent Transportation Systems (4): 267-75. [doi: 10.1109/TITS.2004.837816].

[16] Chen, B., Cheng, H. H., and Palen, J. 2006. "Mobile-C: A Mobile Agent Platform for Mobile C/C++ Agents."
Journal of Software: Paactice and Experience 36: 1711-33. [doi: 10.1002/spe.742].

[17] Ibarra, S., Castán, J., and Laria, M. 2014. "Optimizing Urban Traffic Control Using a Rationl Agent.” Journal of Zhejiang University SCIENCE C. 15 (12): 1123-37. ISSN: 1869-1951. [doi: 10.1631/jzus.C1400037]. 